\title{
Correction to: C
}

\section{Correction to: Chapter 3 in: L. K. Golightly et al., Renal Pharmacotherapy, https://doi.org/10.1007/978-3-030-58650-8_3}

The following changes have been made in the original version of chapter " $\mathrm{C}$ ":

- On page 154, the first listed reference by Benzman et al has been replaced with the following reference:

"Avycaz® injection [package insert]. Madison NJ: Allergan USA Inc; 2020."

- On page 155, dosage interval or frequency tabulation had incorrect numerical dosage interval or frequency, which has now been corrected in the updated version. 